\title{
CS Research Square \\ The potential genes mediate the pathogenicity of allogeneic CD4+T cell in aGVHD mouse model
}

\author{
Zheng-Yu Yu \\ Peking University First Hospital \\ Chen-Chen Qin \\ Peking University First Hospital \\ Min Cao \\ Peking University First Hospital \\ Xiao-Ya He \\ Peking University First Hospital \\ Han-Yun Ren \\ Peking University First Hospital \\ Hui-Hui Liu ( $\nabla$ huihuiliu@bjmu.edu.cn ) \\ Peking University First Hospital
}

\section{Research Article}

Keywords: bone marrow transplantation, chemokines, acute graft-versus-host disease

Posted Date: February 11th, 2021

DOl: https://doi.org/10.21203/rs.3.rs-208812/v1

License: (c) (i) This work is licensed under a Creative Commons Attribution 4.0 International License.

Read Full License 


\section{Abstract}

Acute graft-versus-host disease (aGVHD) remains a significant and severe complication of allogeneic hematopoietic stem cell transplantation (allo-HSCT). Due to the occurrence of aGVHD, allo-HSCT significantly increases the mortality rate compared with autologous hematopoietic stem cell transplantation (auto-HSCT). As a transcriptome sequencing technique, ribonucleic acid sequencing (RNA-Seq) is used to study alternative splicing and other forms of alternative isoform expression. Major histocompatibility complex (MHC)-matched and MHC-mismatched mouse bone marrow transplant (BMT) models were built to detect the difference of CD4+ lymphocyte in different tissues based on RNAseq analysis. Four critical pathways were identified by enrichment analysis, including a lymphoid and a non-lymphoid cell, chemokine receptor binding chemokine, cytokine and cytokine receptor interaction pathway. At the same time, 11 novel hub genes that are most likely to participate in immunoregulatory and enriched pathways were identified, which were further validated by qRTPCR. Besides, Cxcl7 was verified at the protein level. In summary, we revealed that DEGs and pathways related to immunoregulation played a vital role in the onset of aGVHD. These findings may provide some new clues for probing the pathogenesis and treatment of aGVHD.

\section{Introduction}

Allogeneic hematopoietic stem cell transplantation (allo-HSCT) is an effective treatment for various haematological diseases of which blood cells are unavailable in unmodified autologous transplants. Acute graft-versus-host disease (aGVHD) is one of the significant complications of allo-HSCT. The interaction between $\mathrm{T}$ cell receptors, antigen-MHC complexes, costimulatory molecules and cytokines activate the donor $\mathrm{T}$ cells at the early stage. Activation of $\mathrm{T}$ cells regulates gene expression and triggers proliferation, differentiation, and effector functions. The activated effector $\mathrm{T}$ cells migrate to target tissues and recruit more effector cells, resulting in damage to the lung, gut, and liver ${ }^{1}$. Alloreactive T cells proliferate and differentiate into subsets of T cells, including Th1, Th2, Th17, and others in peripheral lymphoid tissues, which are considered as the major detrimental factors during the pathogenesis of aGVHD. Th1 cells secreting IFN-y are treated as the main drivers of the onset and progress of aGVHD ${ }^{2}$. In these stages, $T$ cells in the transplant are activated under the stimulation of inflammatory environment and allogeneic antigen, clonally proliferate and finally different into helper Th (CD4 ${ }^{+} \mathrm{T}$ cells) cells or cytotoxic Tc (CD8 ${ }^{+} \mathrm{T}$ cells) cells ${ }^{3}$. Among all T cells, donor-derived $\mathrm{CD} 4^{+} \mathrm{T}$ cells and secreted cytokines are particularly vital in the pathogenesis and regulation of aGVHD. Studies have shown that the regulation of donor-derived $\mathrm{CD} 4^{+} T$ cells and the production of some critical cytokines (such as IFN- $\gamma$ ) could relieve the occurrence and development of aGVHD ${ }^{4}$. CD $4^{+} \mathrm{T}$ cells have been studied as the potential treatment targets for GVHD in a large number of clinical trials ${ }^{2}$. However, a clinical study found that peripheral blood stem cell (PBSC) graft containing more mature T cells did not increase the incidence and severity of aGVHD ${ }^{5}$. Meanwhile, we found a phenomenon that the infiltration of activated effector $C D 4^{+} T$ cells in these tissues was different in the aGVHD mouse model. The exact character of activated effector $C D 4^{+} T$ cells in aGVHD remains unclear. In the study, using ribonucleic acid sequencing (RNA-seq), we analyzed 
the gene expressing profiles of $\mathrm{CD} 4^{+} \mathrm{T}$ cells from the spleen, lung, liver in auto-HSCT and allo-HSCT recipient mouse and identified the differentially expressed genes (DEGs) to characterize $C D 4^{+} T$ cells in aGVHD.

\section{Results}

Detect RNA sequence information of $\mathrm{CD}^{+}{ }^{+} \mathrm{T}$ cells in target organs of HSCT-mice. The samples of target organs were excised at day 7 after transplantation and infiltrated by $C D 4^{+} T$ cells (Fig. $1 \mathrm{~A}$ ).CD $4^{+} \mathrm{T}$ in different organs of Balb/c recipient mice had $>92 \%$ purity (Fig.1B). The percent of $C D 44^{+} \mathrm{CD} 62 \mathrm{~L}^{\text {low }}$ effector cells was about $90 \%$ in allo-HSCT mouse (Fig. 1C). Clean reads that met the quality control criteria of pre-processing constituted $>96 \%$ of all reads in all samples (Fig.S1A). The percentage of reads mapped in the exon region above $94 \%$ (Fig. S1B). The error rate of all samples was less than $0.1 \%$ (Fig. S1C). For chain specific libraries, the contents of $A / T$, and $G / C$ were almost the same (Fig. S1D). Boxplots of the expression levels of all genes was shown that no sample errors and outliers in Fig. S2A. The correlation coefficient R2 and PCA analysis indicated the high comparability between groups(Fig.S2B-C). These results suggest high quality of our data with great replicability within groups and comparability between groups.

Identification DEGs of $\mathrm{CD}^{+} \mathrm{T}$ cells from HSCT-mice. In order to detect the impact of xenoantigen and intertissue microenvironment on donor $\mathrm{CD} 4^{+} \mathrm{T}$, the spleen $\mathrm{CD} 4^{+} \mathrm{T}$ cells of allogeneic transplanted mice without the influence of xenoantigen were regarded as the control group, and different organs $C D 4^{+} T$ of allogeneic transplanted mice affected by xenoantigen and microenvironment as the analysis group. The DEGs and samples were analyzed by clustering analysis. The results indicated that genes with similar expression patterns were functionally correlated (Fig. 2B). Based on padj $<0.05$ and $\log _{2}$ (Fold Change) $>1$ as shown in the volcano map (Fig. 2A), a total 2996 (1673 upregulated and 1323 downregulated), 3955 (2131 upregulated and 1824 downregulated) and 5252 (3289 upregulated and 1966 downregulated) DEGs were identified in Allo-Spleen vs Auto-Spleen, Allo-Liver vs Auto-Spleen and Allo-Lung vs AutoSpleen. We further explored the group-specific and overlapped DEGs in these groups. There are 1188 overlapped DEGs and 746, 2661, and 1431 group-specific DEGs identified in Allo-Spleen, Allo-Lung, and Allo-Liver, respectively (Fig. 2C).

Analysis of immune-related pathways in HSCT-mice. Gene functions of DEGs were annotated and classified from three aspects: Biological Process (BP), Cellular Component (CC), and Molecular Function (MF). There were $380 \mathrm{BP}$ terms, $14 \mathrm{CC}$ terms, $17 \mathrm{MF}$ terms in Allo-Spleen vs Auto-Spleen, $851 \mathrm{BP}$ terms, $48 \mathrm{CC}$ terms, $141 \mathrm{MF}$ terms in Allo-Liver vs Auto-Spleen and 1339 terms, $47 \mathrm{CC}$ terms, $93 \mathrm{MF}$ terms in AlloLung vs Auto-Spleen statistically significantly enriched in DEGs, respectively. The top $10 \mathrm{GO}$ terms were presented in Fig. 3. It should be noted that 6 BP terms (regulation of leukocyte activation, leukocyte chemotaxis, regulation of lymphocyte activation, regulation of chemotaxis, chemotaxis and leukocyte migration) and $3 \mathrm{MF}$ terms (cytokine activity, chemokine activity and cytokine receptor binding) were distinguished to be associated with immunoregulation (Fig. 3). 
Of the top 10 most significantly enriched pathways were shown using KEGG and Reactome (Fig. 4A-C). Gene set enrichment analysis (GSEA) revealed 2 KEGG pathway (The chemokine signaling pathway, cytokine and cytokine receptor interaction) and 2 Reactome pathways (chemokine receptors binding chemokines, lymphoid and a non-lymphoid cell) belonged to immunoregulation in GVHD (Fig. 4D-F).

Potential immunoregulatory genes in DEGs. To gain insight into the relationships of DEGs, we constructed a PPI network with high confidence interactions using STRING and Cytoscape. Table 2 listed the top ten hub genes, a total of 60 hub genes, from each group identified by the CytoHubba plugin. Eleven out of 60 hub genes were associated with immunoregulation and haven't been studied in GVHD, including Rgs1, Fpr2, Ppbp (CxCl7), S1pr3, Npy, Hebp1, Kng1, Plg, Cxcl16, Ccl9 and Cxcl3. The PPI network among the 11 genes was constructed (Fig. S4A). According to MCC, CxCl16, Kng1 and CxCl7 were considered as hub genes (Fig. S4B). Moreover, we applied the MCODE plugin to analyze PPIs to predict significant protein complexes. One significant module was identified in the DEGs regulatory network, which contained 8 genes, including $\mathrm{CxCl} 3, \mathrm{Npy}, \mathrm{CxCl16}$, Kng1, Ccl9, S1pr3, Fpr2 and $\mathrm{CxCl7}$ (Fig. S4C).

Validation of the sequence results in vitro. To confirm the credibility of the DEGs identified from RNA-Seq, we performed qRT-PCR to verify the expression level of the DEGs. The CD $4^{+} T$ cells activated by MLR and cytokines (Fig. 5A, B) were obtained by FACS-sorted. Four related hub genes were verified by activated $\mathrm{CD} 4^{+} \mathrm{T}$ cells through MLR and cytokines stimulated (Fig.5C-D). These genes showed consistent results as in RNA-seq.

Validation of the sequence results in vivo. The validation data GSE126518 obtained from the GEO database was further used to verified the results (Fig. 6A). We also selected the 11 novel hub genes that were significantly differentially expressed among liver, lung and spleen. As shown in Fig. 6B, most of the

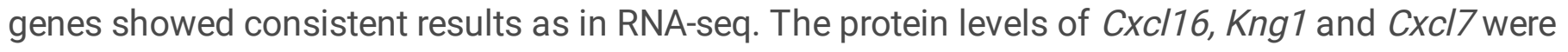
verified by histological research and immunofluorescence staining. The results showed that the expression level of $\mathrm{CxCl} 7$ in Auto-Spleen was higher than that of Allo-Spleen (Fig.6C-D). However, Kng1 protein and $\mathrm{Cxcl} 16$ protein were almost undetectable in Auto-Spleen and Allo-Spleen and there was no statistical difference about the expression level of $C x C / 16$ and $K n g 1$ between Auto-Spleen and Allo-Spleen (the data was not shown). Moreover, it was found that the expression level of $\mathrm{CxCl}$ w was also elevated in Auto-Liver and Auto-Lung (Fig.S4). In conclusion, the results basically verified the reliability of result.

\section{Discussion}

Acute GVHD is induced by numerous graft-derived immunocytes, including the naive $\mathrm{CD} 4^{+} \mathrm{T}$ cells from donors which can differentiate and exert cytotoxicity on multiple organ systems of the allogeneic hematopoietic stem cell transplantation recipient ${ }^{2}$. However, whether the effector CD ${ }^{+} T$ expressed differently among different organs was uncertain. RNA-Seq, a method of comprehensive transcriptome profiling, generates deep sequencing data for the direct quantification of transcripts by next-generation sequencing (NGS) technologies ${ }^{6}$. This study leveraged integrative transcriptome analysis to explore potential molecular mechanisms involved in $\mathrm{CD} 4^{+} \mathrm{T}$ cells in different target organs from auto-HSCT and 
allo-HSCT aGVHD mouse models. We identified significant DEGs and enriched pathways, including chemokine receptor binding chemokine, a lymphoid and a non-lymphoid cell and cytokine and cytokine receptor interaction pathway. Eleven novel genes that highly possibly participated in immunoregulatory of the pathogenesis of aGVHD were also identified. In the PPI network, two significant protein complexes containing DEGs regulators were detected.

RNA-Seq was not free of biases. Results could be affected by differences in fragment size, transcript length and differences in GC content ${ }^{7}$. In this study, to ensure the high quality and creditability of the data and results, we collected high purity $\mathrm{CD} 4^{+} \mathrm{T}$ cells $(>92 \%), \mathrm{CD} 44^{+} \mathrm{CD} 62 \mathrm{~L}^{\text {low }}$ effector cells (about $90 \%$ ) in allo-HSCT mouse, and applied quality control filters in the preprocessing of the raw-read before identification of DEGs. Boxplots, correlation heatmap and PCA plots of the gene expression FPKM values provided further evidences for the high quality and creditability of our data.

Organs have unique structures, physiological functions and tissue microenvironments, thereby forming local immune environments that may be closely related to the occurrence and development of GVHD associated with the organs. There are few studies on the local immune environments of target organs in GVHD. Spleen is the largest peripheral lymphatic organ in the human body, harboring various types of lymphocytes, mainly B and T cells. These cells in spleen exert immune responses when the body is invaded by pathogens. To further explore the immunopathological mechanism of GVHD, we studied the DEGs with a focus on hub genes with immune regulation functions from the spleen, liver, and lung in the allo-HSCT mouse model compared with auto-spleen.

We identified 1188 overlapped DEGs and 746, 2661, and 1431 organ-specific DEGs by CD $4^{+} \mathrm{T}$ in AlloSpleen, Allo-Lung and Allo-Liver compared Auto-Spleen. Many DEGs were found associated with GVHD in previous studies, such as CD34 and CD33 ${ }^{8,9}$. We also identified many novel DEGs that are firstly reported to be associated with aGVHD in our study. Pathway enrichment analysis and PPI networks are great ways to extract main information from a large list of genes thus were often used in transcriptome analysis. Due to the effect on the relevant pathway and regulation of other genes, hub genes in PPI networks provide promising targets and research hotspots in the biological process. We identified 60 hub genes in PPI networks of DEGs, including 11 novel genes that were not reported in previous GVHD studies. Eleven of 11 novel genes were later confirmed with qRT-PCR.

We observed a significant portion of the hub genes were chemokine genes, especially the upregulated DEGs in the spleen and lung from allogenic recipient mice. Reactome pathway analysis of the DEGs also highlighted the chemokine receptor binding chemokine pathway. One main function of chemokines is to induce immune cells to enter the site of infection during the immune response ${ }^{10}$. In aGVHD model, the activated effector $T$ cells migrate to target tissues and organs to recruit more effector cells, resulting in tissue and organ damage. The role of chemokines in GVHD has been studied by our team and many other researches ${ }^{11,12}$. We found that the increased expression of $\mathrm{CxCl} 3$ was consistent with the enrichment of the pathway. $\mathrm{CxC} / 3$ increases the activity toward human granulocytes ${ }^{13}$. Other nonchemokine genes, such as Rgs 1 and Fpr2, can increase the response of chemokines and regulate 
inflammatory mediators and enhance immune regulation properties ${ }^{14,15}$. In this study, we identified more chemokine genes that were not previously reported in GVHD, especially $\mathrm{CxCl} / \mathrm{K}, \mathrm{Kng} 1$ and $\mathrm{CxC} / 16$ which could be investigated in high priority in future studies.

Our study found that cytokine and cytokine receptor interaction pathways are significantly enriched in DEGs by KEGG pathway analysis. Cytokines have important immunoregulatory functions during immune responses. At the early stage of GVHD, cytokines recruit various subsets of immune cells to immune context. Our study found increased expression of Npy in the spleen of auto-HSCT. Npy can regulate the expression of Interleukin-4 (IL-4) ${ }^{16}$, a leukocyte chemotactic activating cytokine that guide the differentiation of naive $\mathrm{CD} 4^{+} \mathrm{T}$ cells into Th2 cells ${ }^{17}$. The high expression of Npy from auto-HSCT promoted DCs to polarize Th2 differentiation by up-regulation of IL-10 ${ }^{18}$. Moreover, Npy could decrease the production and release of IL- 6 from splenic macrophages ${ }^{19}$, which may be related to the reduction of the severity of the disease caused by the inhibition of cytokine release.

Reactome enriched pathway analysis also discovered a lymphoid and non-lymphoid cell pathway from DEGs. Among the hub genes, the Plg gene, a liver-specific gene ${ }^{20}$, was found highly expressed in $C D 4^{+} T$ cell from the liver of aGVHD model. Mitchell et al. ${ }^{21}$ revealed that the $\mathrm{Plg}$ promoted monocyte proliferation in the inflammation response. However, its function in GVHD is unknown. Epithelial cells can collaborate with immune cells to regulate the innate and adaptive response in several tissue types ${ }^{22}$. It is possible that genes like $\mathrm{Plg}$ might be involved in the lymphoid and non-lymphoid cells immunoregulatory interaction. Regulation of these genes with the characteristics of immune regulation and tissue-specific expression could be used to decrease the influence of activated $\mathrm{CD} 4^{+} \mathrm{T}$ cells to infiltrate and damage organs in the future.

\section{Conclusion}

This is the first report of mRNA transcript profiling of effector $C D 4^{+} T$ cells from spleen, liver, and lung in aGVHD mouse model. We identified significant DEGs, including novel genes, from spleen, liver and lung from allo-HSCT aGVHD model. Pathway analysis and PPI networks of the DEG highlighted the chemokine receptor binding chemokine pathway and the lymphoid and non-lymphoid cells immunoregulatory interaction in the process of GVHD. We identified 11 hub genes that were novel in aGVHD, 10 of which were confirmed with qRT-PCR. Our findings enhanced the understanding of the mechanism of aGVHD target organ damage and provided a list of genes in high priorities for future studies. More such studies will eventually provide evidences for pre-clinical trials using cellular and animal models and accelerate the development of new methods for clinical therapy.

\section{Materials And Methods}

Mice. Female C57BL/6 $(\mathrm{H}-2 \mathrm{~b})$ and male Balb/c $(\mathrm{H}-2 \mathrm{~d})$ mice were purchased from the Animal Experiment Center of Peking University Health Science Center. C57BL/6 mice, 8-10 weeks of age with a bodyweight of 
19-21g each, were donors for bone marrow hematopoietic stem cells and spleen lymphocytes. Balb/c mice, 10-12 weeks of age with a bodyweight of 20-23g each, were recipients. Mice were fed water containing gentamicin for seven days post-transplantation. Mice were bred under specific pathogen-free conditions at the animal breeding facilities at Peking University Health Science Center. All experimental protocols were approved by the Animal Care and Use Committee of Peking University Health Science Center. Study design was illustrated in Fig. S1 for the experimental and analysis scheme.

aGVHD induction in a murine model. The method of induction of aGVHD using radiation-exposed mice and allogeneic bone marrow transplants was described previously ${ }^{11,12,23}$. Briefly, bone marrow cells were acquired from C57BL/ 6 mice. Splenocytes were obtained from C57BL/ 6 mice and Balb/c by Ficoll gradient centrifugation. Auto-Balb/c and allo-Balb/c recipient mice were irradiated with 7.5Gy of total body irradiation (TBI) and intravenously injected with $1 \times 10^{7} \mathrm{BM}$ cells and $1 \times 10^{7}$ splenic mononuclear cells. aGVHD mouse model was then evaluated by a clinical score including activity, skin integrity, weight loss, posture, and fur texture every other day for one month.

T lymphocyte enrichment. $C D 4^{+} \mathrm{T}$ cells were negatively selected from liver, lung, and spleen of Balb/c recipient mice using Mouse $C D 4^{+} T$ cell isolation kit (Biolegend, San Diego, CA, USA) according to the manufacturer's instructions. CD4 $4^{+}$lymphocytes were prepared at a concentration of $5 \times 10^{6} / \mathrm{mL}$. The purity of enriched populations was $>90 \%$ as assessed by flow cytometry.

Antibodies and flow cytometry. The following anti-mouse antibodies from Biolegend (Cal:100421, 103043, 104411, US) were used in flow cytometry: CD4-PE/Cy7, CD44-BV510, CD62L-APC. Spleens, lungs, and livers were excised and gently pressed through a cell strainer $(70 \mu \mathrm{m})$ on day seven after transplantation. Liver and lung infiltrating lymphocytes were isolated using Percoll (Living, Beijing, China). Single-cell suspensions were blocked by incubating with an anti-Fc receptor antibody for 10 minutes on ice. Cells were then labelled with fluorescently conjugated antibodies at $4^{\circ} \mathrm{C}$ for $30 \mathrm{~min}$, followed by washes with cold PBS twice before membrane molecule analysis by flow cytometer. Cells were sorted by using the BD Symphony S6, and gating was done using the BD FACSDiva software (Becton,Dickinson Biosciences).

RNA preparation. Total RNA was extracted from $\mathrm{CD}^{+}{ }^{+} \mathrm{T}$ cells isolated from the liver, lung, and spleen using a Trizol reagent (Thermo Fisher, Waltham, MA, USA), Qubit ${ }^{\circledR}$ RNA Assay Kit in Qubit ${ }^{\circledR} 2.0$ Fluorometer (Life Technologies, CA, USA). RNase free DNase I (Invitrogen, Carlsbad, CA, USA) was used to digest potential genomic deoxyribonucleic acid (DNA). The digested products were then purified using magnetic beads (Axygen, Union City, CA, USA) to eliminate residual DNA and DNase. Concentration of the extracted RNA was analyzed with a Qubit ${ }^{\circledR}$ RNA Assay Kit in Qubit ${ }^{\circledR} 2.0$ Fluorometer (Life Technologies, CA, USA). RNA purity and integrity were assessed using the NanoPhotometer ${ }^{\circledR}$ spectrophotometer (IMPLEN, CA, USA) and the RNA Nano 6000 Assay Kit of the Bioanalyzer 2100 system (Agilent Technologies, CA, USA). Total RNA of all samples had concentration $\geq 200 \mathrm{ng} / \mathrm{mL}$, mass $\geq 10 \mathrm{mg}$, and RNA integrity number $(\mathrm{RIN}) \geq 8.0$. 
Complementary DNA (cDNA) library construction. The poly-T oligo-attached magnetic beads were used to purify mRNA from total RNA. Fragmentations were carried out using divalent cations under elevated temperature in First Strand Synthesis Reaction Buffer (5x). The cleaved RNA fragments served as the templates for the synthesis of the first-strand CDNA using random hexamer primer and M-MuLV reverse transcriptase (RNaseH-). The second strand cDNA was synthesized using DNA polymerase I and RNase $\mathrm{H}$. The double-stranded cDNA was then degraded to blunt ends using exonuclease/polymerase. After adding a terminal $A$ at the $3^{\prime}$ ends in DNA fragments, library fragments were purified using the AMPure XP system and the cDNA fragments of 250 300 bp in length were selected (Beckman Coulter, Beverly, USA). The sequencing adaptors and the cDNA fragments were mixed and ligated at $37^{\circ} \mathrm{C}$ for $15 \mathrm{~min}$. Polymerase chain reaction (PCR) was performed with Phusion high-fidelity DNA polymerase, universal PCR primers, and index $(X)$ primer. The integrity and size of the product were verified on an Agilent 2100 Bioanalyzer (Agilent Technologies, Santa Clara, CA, USA). RNA-Seq data processing and identification of DEGs. cDNA libraries of CD $4^{+} \mathrm{T}$ cells from auto-HSCT and allo-HSCT model were sequenced at Novogene Bioinformatics Technology Co. (Beijing, China). Paired-end sequencing were performed on an Illumina HiseqTM 4000 platform (Illumina, San Diego, CA, USA). FastQC software was used to preprocess raw reads to remove reads containing PCR duplicates, adapter, ploy-N and low-quality reads. In this step, Q20, Q30 and GC content of the clean reads were calculated. All the subsequent analyses were based on the clean data with high quality.

Gene model annotation files and reference genome were downloaded from genome website (http://apr2018.archive.ensembl.org/Mus_musculus/Info/Annotation). Hisat2 v2.0.5 was used to build the index of the reference genome and to match paired-end clean reads with the reference genome. The sequencing process possibly has machine errors, and the inspection of the error rate can reflect the quality of sequencing data. The error rate was obtained through conversion of the Phred score of the sequencing base through the formula Qphred $=-10 \log 10(e)$, where $E$ is the base error rate ${ }^{24}$.

Fragments per kilobase per million reads (FPKM) was used to calculate the transcript expression levels ${ }^{25}$. The DESeq2 R package (1.16.1) was used to identify differentially expressed genes (DEGs) in CD $4^{+} T$ cells from different organs between autologous and allogeneic hematopoietic stem cell transplantation mice ${ }^{26}$.

According to the manufacturer's instructions, the clustering of the index-coded samples was carried out on a cBot Cluster Generation System by TruSeq PE Cluster Kit v3-cBot-HS (Illumia). The union of DEGs of all groups are taken as the DEGs sets. In the multi-group experiments, the clustering analysis of DEGs sets was performed to gather genes with similar expression patterns.

GO and pathway enrichment analysis. All DEGs were mapped to terms in the Gene Ontology (GO) databases for functional enrichment analysis. $\mathrm{GO}$ terms. The padj $<0.05$ were considered significantly enriched. GO term was classified into three subgroups: biological process (BP), cellular component (CC), and molecular function (MF). Pathways with padj $<0.05$ for DEGs were further annotated by the Kyoto Encyclopedia of Genes and Genomes (KEGG) and the Reactome automatic annotation server ${ }^{27,28}$. 
Genome enrichment analysis of differentially expressed genes in immune-related pathways was performed using GEAS $₫$ Gene Set Enrichment Analysis $\mathbb{2}^{29}$.

PPI network construction analysis. STRING (Search Tool for the Retrieval of Interacting Genes) was applied to predict the protein-protein interaction (PPI) network for DEGs ${ }^{30}$. The Cytoscape software was used to visualize and analyze the PPI network (confidence score $\geq 0.4$, the maximum number of interactors $=0)^{31}$. The CytoHubba plugin from the Cytoscape was applied to look for hub genes according to the Maximal Clique Centrality $(\mathrm{MCC})^{32}$. The intuition behind MCC is that essential proteins tend to be clustered in a yeast protein-protein interaction network. The Molecular Complex Detection (MCODE) plugin was applied to perform the gene network functional module analysis. The method is based on vertex weighting based on local neighborhood density and outward traversal of local dense seed protein, and the dense regions are separated according to the given parameters ${ }^{33}$.

Validation of gene expression by qRT-PCR. Real-time quantitative reverse transcription polymerase chain reaction (qRT-PCR) was performed to validate the expression levels of 11 DEGs related to immunoregulation. The murine gene GAPDH served as an endogenous reference. The primers for all genes were presented in Table 1. Total RNA was extracted and used as a template to be reversely transcribed into single-stranded cDNA using Revert Aid first strand cDNA Synthesis Kit (Thermo Fisher, Waltham, MA, USA). The ABI Prism 7500 PCR system was used for real-time monitoring of SYBR Green dye (Life, Foster City, CA, USA) integrated into PCR products. Reactions were carried out at following conditions: initial denaturation at $50^{\circ} \mathrm{C}$ for $2 \mathrm{~min}$ and then $95^{\circ} \mathrm{C}$ for $10 \mathrm{~min}$, followed by 40 cycles of reaction at $95^{\circ} \mathrm{C}$ for $15 \mathrm{~s}$ and $60^{\circ} \mathrm{C}$ for $60 \mathrm{~s}$. Each sample had three technical replicates (3 independent wells with the same template) to ensure the precision of the quantification. The $2^{-\Delta \Delta C t}$ method was used for gene expression.

Cell stimulation and expansion. T cells $\left(5 \times 10^{5} / \mathrm{mL}\right)$ from spleen of Balb/c or C57BL/ 6 mice, separated by the $C D 4^{+} T$ cell negative selection magnetic cell sorting, were stimulated with anti-CD3/CD28 antibodies coated plates at $500 \mu \mathrm{L}$ of $2 \mathrm{ug} / \mathrm{mL}$ each (PeproTech, USA) for 48 hours.

Mixed lymphocyte reaction. Spleen was isolated from mice, ground into individual cells, and treated with $50 \mu \mathrm{g} / \mathrm{mL}$ mitomycin C (Sigma,USA) for $30 \mathrm{~min}$ at $37^{\circ} \mathrm{C}$. Following washed three times by PBS, responder $\left(0.5 \times 10^{6} \mathrm{C} 57 \mathrm{BL} / 6 \mathrm{CD} 4^{+} \mathrm{T}\right.$ cells $)$ and stimulator $\left(0.2 \times 10^{6} \mathrm{Balb} / \mathrm{c}\right.$ spleen cells $)$ were co-cultured in $200 \mu \mathrm{L}$ RPMI 1640 culture medium containing $10 \% \mathrm{FBS}, 100 \mu \mathrm{g} / \mathrm{mL}$ streptomycin and $100 \mu \mathrm{g} / \mathrm{mL}$ penicillin. After co-culture for $72 \mathrm{~h}$, cells were collected and tested for $\mathrm{T}$ cell activation. Activated C57BL/ $6 \mathrm{CD} 4^{+} \mathrm{T}$ cells $\left(\mathrm{H} 2 \mathrm{~KB}^{+} \mathrm{CD} 4^{+} \mathrm{CD} 44^{+}\right)$were separated by FACS (fluorescence activated cell sorting)-sorted.

Histological research. Mice in each group were sacrificed on day 7 after transplantation. The liver, lung and spleen tissues were fixed in $10 \%$ buffered formalin, and embedded in paraffin blocks for subsequent hematoxylin and eosin staining. Histologic changes were graded and scored by a pathologist blinded to the clinical status of the mice. Four items were assessed as described previously ${ }^{34}$. To determine the infiltration of T cells in the tissues of post-HSCT mice, immunohistochemical analysis was performed for 
staining with primary antibody anti-CD4 (1:20, Servicebio, Wuhan, China) and anti-Cxcl7 (1:20, Abcam, Cambridge, UK)as well as the appropriate horseradish peroxidase-conjugated secondary antibody (1:50, Servicebio, Wuhan, China) on the liver, lung and spleen tissues of recipient mice at 7 days posttransplantation according to the manufacturer's instructions.

Immunofluorescence Staining. The spleen tissues were fixed with acetone. Immunofluorescent staining was performed using primary antibody anti-CD4 (1:200, Servicebio, Wuhan, China), anti-Cxcl7 (1:2000, Abcam, Cambridge, UK), and secondary antibody conjugated to FITC (1:500, Servicebio, Wuhan, China) in low light conditions. Nuclei were stained with DAPI (Servicebio, Wuhan, China). Confocal microscopy was used to image for immuno-stained slides.

Statistical analysis. DEGs were identified by the DESeq2 R package $\mathrm{e}^{35}$. The significance was calculated by the hypothesis testing probability (p-value), and corrected for multiple testing using Benjaminihochburg method (also known as FDR value). Significant DEGs were defined to meet the following two requirements: (i) more than two folds change in expression; (ii) padj $<0.05$. The fold change of each gene was calculated by comparing the standardized control read counts of induced CD4 ${ }^{+}$. $\log _{2}$ scale of fold change was used for convenience. $\log _{2}$ (fold change) $>1$ means $>$ two folds change. For other comparisons in this study, statistically significant differences were calculated by the Student's t-test and ANOVA. The $p<0.05$ was considered significant.

\section{Declarations}

\section{Conflict of interest}

The authors declare no conflict of interest.

\section{Acknowledgement \& funding}

This study was supported by grant from The National Natural Science Foundation of China (NSFC) (Grant Number 81600144 and 81970160) and grant from Research Foundation of Peking University First Hospital.

\section{Author Contributions Statement}

ZY contributed conception and design of the study, performed the most experiments of this study and wrote the paper. CQ performed the MLR assay and part of aGVHD models. CM and XY helped to establish aGVHD mouse models. HL performed part of flow cytometric analysis and qPCR experiments. HY and HL contributed conception and design of the study. All authors contributed to manuscript revision, read and approved the submitted version.

\section{Conflict of interest statement}

The authors declared that they have no competing interests. 


\section{References}

$1 \quad \mathrm{Yu}, \mathrm{Y}$. et al. Prevention of GVHD while sparing GVL effect by targeting Th1 and Th17 transcription factor T-bet and RORyt in mice. Blood 118, 5011-5020, doi:10.1182/blood-2011-03-340315 (2011).

2 Blazar, B. R., Murphy, W. J. \& Abedi, M. Advances in graft-versus-host disease biology and therapy. Nat Rev Immuno/ 12, 443-458, doi:10.1038/nri3212 (2012).

3 Coghill, J. M. et al. Effector CD4+ T cells, the cytokines they generate, and GVHD: something old and something new. Blood 117, 3268-3276, doi:10.1182/blood-2010-12-290403 (2011).

$4 \quad$ Reddy, P. et al. Interleukin-18 regulates acute graft-versus-host disease by enhancing Fasmediated donor T cell apoptosis. J Exp Med 194, 1433-1440, doi:10.1084/jem.194.10.1433 (2001).

$5 \quad$ Guardiola, P. et al. Retrospective comparison of bone marrow and granulocyte colonystimulating factor-mobilized peripheral blood progenitor cells for allogeneic stem cell transplantation using HLA identical sibling donors in myelodysplastic syndromes. Blood 99, 4370-4378, doi:10.1182/blood.v99.12.4370 (2002).

6 Kyttälä, A. et al. Genetic Variability Overrides the Impact of Parental Cell Type and Determines iPSC Differentiation Potential. Stem cell reports 6, 200-212, doi:10.1016/j.stemcr.2015.12.009 (2016).

7 Roberts, A., Trapnell, C., Donaghey, J., Rinn, J. L. \& Pachter, L. Improving RNA-Seq expression estimates by correcting for fragment bias. Genome biology 12, R22, doi:10.1186/gb-2011-12-3-r22 (2011).

$8 \quad$ Wang, K. et al. Early myeloid-derived suppressor cells (HLA-DR(-)/(low)CD33(+)CD16(-)) expanded by granulocyte colony-stimulating factor prevent acute graft-versus-host disease (GVHD) in humanized mouse and might contribute to lower GVHD in patients post allo-HSCT. Journal of hematology \& oncology 12, 31, doi:10.1186/s13045-019-0710-0 (2019).

9 Ono, R. et al. Co-activation of macrophages and T cells contribute to chronic GVHD in human IL6 transgenic humanised mouse model. EBioMedicine 41, 584-596, doi:10.1016/j.ebiom.2019.02.001 (2019).

10 Palomino, D. C. \& Marti, L. C. Chemokines and immunity. Einstein (Sao Paulo, Brazil) 13, 469-473, doi:10.1590/s1679-45082015rb3438 (2015).

11 Tang, B. et al. CCR5 blockade combined with cyclosporine A attenuates liver GVHD by impairing T cells function. Inflammation research : official journal of the European Histamine Research Society ... [et al.] 65, 917-924, doi:10.1007/s00011-016-0974-6 (2016). 
Miao, S. et al. CXCR3 blockade combined with cyclosporine A alleviates acute graft-versus-host disease by inhibiting alloreactive donor T cell responses in a murine model. Molecular immunology $\mathbf{9 4}$, 82-90, doi:10.1016/j.molimm.2017.12.010 (2018).

13 Geiser, T., Dewald, B., Ehrengruber, M. U., Clark-Lewis, I. \& Baggiolini, M. The interleukin-8-related chemotactic cytokines GRO alpha, GRO beta, and GRO gamma activate human neutrophil and basophil leukocytes. The Journal of biological chemistry 268, 15419-15424 (1993).

14 Han, J. I., Huang, N. N., Kim, D. U. \& Kehrl, J. H. RGS1 and RGS13 mRNA silencing in a human B lymphoma line enhances responsiveness to chemoattractants and impairs desensitization. Journal of leukocyte biology 79, 1357-1368, doi:10.1189/jlb.1105693 (2006).

15 Gaudin, A., Tolar, M. \& Peters, O. A. Lipoxin A(4) Attenuates the Inflammatory Response in Stem Cells of the Apical Papilla via ALX/FPR2. Scientific reports 8, 8921, doi:10.1038/s41598-018-27194-7 (2018).

16 Wheway, J., Herzog, H. \& Mackay, F. NPY and receptors in immune and inflammatory diseases. Current topics in medicinal chemistry 7, 1743-1752, doi:10.2174/156802607782341046 (2007).

17 Junttila, I. S. Tuning the Cytokine Responses: An Update on Interleukin (IL)-4 and IL-13 Receptor Complexes. Frontiers in immunology 9, 888, doi:10.3389/fimmu.2018.00888 (2018).

18 Buttari, B. et al. Neuropeptide $\mathrm{Y}$ induces potent migration of human immature dendritic cells and promotes a Th2 polarization. FASEB journal : official publication of the Federation of American Societies for Experimental Biology 28, 3038-3049, doi:10.1096/fj.13-243485 (2014).

19 Straub, R. H. et al. Neuropeptide Y cotransmission with norepinephrine in the sympathetic nervemacrophage interplay. Journal of neurochemistry 75, 2464-2471, doi:10.1046/j.14714159.2000.0752464.x (2000).

20 Yue, F. et al. A comparative encyclopedia of DNA elements in the mouse genome. Nature 515, 355-364, doi:10.1038/nature13992 (2014).

21 Mitchell, J. W., Baik, N., Castellino, F. J. \& Miles, L. A. Plasminogen inhibits TNFalpha-induced apoptosis in monocytes. Blood 107, 4383-4390 (2006).

22 Barrios De Tomasi, J., Opata, M. M. \& Mowa, C. N. Immunity in the Cervix: Interphase between Immune and Cervical Epithelial Cells. Journal of immunology research 2019, 7693183, doi:10.1155/2019/7693183 (2019).

23 Yuan, J., Ren, H. Y., Shi, Y. J. \& Liu, W. Prophylaxis of acute graft-versus-host disease by CCR5 blockade combined with cyclosporine A in a murine model. Inflamm Res 64, 137-144, doi:10.1007/s00011-014-0793-6 (2015). 
24 Mortazavi, A., Williams, B. A., McCue, K., Schaeffer, L. \& Wold, B. Mapping and quantifying mammalian transcriptomes by RNA-Seq. Nature methods 5, 621-628, doi:10.1038/nmeth.1226 (2008).

25 Trapnell, C., Pachter, L. \& Salzberg, S. L. TopHat: discovering splice junctions with RNA-Seq. Bioinformatics 25, 1105-1111, doi:10.1093/bioinformatics/btp120 (2009).

26 Anders, S., Reyes, A. \& Huber, W. Detecting differential usage of exons from RNA-seq data. Genome Res 22, 2008-2017, doi:10.1101/gr.133744.111 (2012).

27 Kanehisa, M. \& Goto, S. KEGG: kyoto encyclopedia of genes and genomes. Nucleic Acids Res 28, 27-30, doi:10.1093/nar/28.1.27 (2000).

28 Fabregat, A. et al. The Reactome Pathway Knowledgebase. Nucleic Acids Res 46, D649-d655, doi:10.1093/nar/gkx1132 (2018).

29 Subramanian, A. et al. Gene set enrichment analysis: a knowledge-based approach for interpreting genome-wide expression profiles. Proc Natl Acad Sci U S A 102, 15545-15550, doi:10.1073/pnas.0506580102 (2005).

$30 \mathrm{Ni}$, M. et al. Identification of Candidate Biomarkers Correlated With the Pathogenesis and Prognosis of Non-small Cell Lung Cancer via Integrated Bioinformatics Analysis. Frontiers in genetics $\mathbf{9}$, 469, doi:10.3389/fgene.2018.00469 (2018).

31 Snider, J. et al. Fundamentals of protein interaction network mapping. Molecular systems biology 11, 848, doi:10.15252/msb.20156351 (2015).

32 Chin, C. H. et al. cytoHubba: identifying hub objects and sub-networks from complex interactome. BMC systems biology 8 Suppl 4, S11, doi:10.1186/1752-0509-8-s4-s11 (2014).

33 Bader, G. D. \& Hogue, C. W. An automated method for finding molecular complexes in large protein interaction networks. BMC bioinformatics 4, 2, doi:10.1186/1471-2105-4-2 (2003).

34 Cooke, K. R. et al. Tumor necrosis factor- alpha production to lipopolysaccharide stimulation by donor cells predicts the severity of experimental acute graft-versus-host disease. The Journal of clinical investigation 102, 1882-1891, doi:10.1172/jci4285 (1998).

35 Varet, H., Brillet-Guéguen, L., Coppée, J. Y. \& Dillies, M. A. SARTools: A DESeq2- and EdgeR-Based R Pipeline for Comprehensive Differential Analysis of RNA-Seq Data. PloS one 11, e0157022, doi:10.1371/journal.pone.0157022 (2016).

\section{Tables}


Table 1 Primers for 11 candidate immunoregulatory genes and Gapdh

\begin{tabular}{|c|l|l|}
\hline Gene & \multicolumn{1}{|c|}{ Forward Primer $\left(5^{\prime} \rightarrow 3^{\prime}\right)$} & \multicolumn{1}{c|}{ Reverse Primer $\left(5^{\prime} \rightarrow 3^{\prime}\right)$} \\
\hline \hline Cxcl7 & CTCACGTTGTTCCTCCTGG & TGGGTCCATGCCATCAGATT \\
\hline S1pr3 & TCAACACTCTTCCCGCAGTC & CCCGGAGAGTGTCATTTCCC \\
\hline $\mathrm{Npy}$ & TAACAAGCGAATGGGGCTGT & TGATGTAGTGTCGCAGAGCG \\
\hline$H e b p 1$ & CCTGTCTACTCACGCGCTTA & GGAGACATCTTCCTTGCCCC \\
\hline$R g s 1$ & TGCCAACCAGACAGGTCAAA & TTCACAGCATCTGAATGCACAA \\
\hline$F p r 2$ & TTCATGGGCCAGGACTTTCG & CACAGACTTCATGGGGCCTT \\
\hline$C x c / 16$ & GGGCTTTGGACCCTTGTCTC & GATCCAAAGTACCCTGCGGT \\
\hline$C c / 9$ & CAGGCCGGGCATCATCTTTA & AGTAGCTGGCAGTTCACACC \\
\hline$C x c / 3$ & CTGCACCCAGACAGAAGTCAT & CCGTTGGGATGGATCGCTTT \\
\hline$P l g$ & TCCGTGGGTTGGATGTTCAG & ATTGTCCGGTCAGCAACCAT \\
\hline $\mathrm{Kng} 1$ & TCCCGACTGTGAAATGCCAA & CCCAGTGTCATATGGTGGGG \\
\hline$G a p d h$ & CTCATGACCACAGTCCATGC & CACATTGGGGGTAGGAACAC \\
\hline
\end{tabular}

Table 2 The top 10 up- and down-regulated hub genes identified in CD4+ T cells from each organ

\begin{tabular}{|c|c|c|}
\hline \multicolumn{2}{|c|}{ Group } & Hub Gene \\
\hline Allo-Spleen vs & Up-regulated & Cxcr2, Ccr1, Ccl5, Rgs1, Cxcl10, Cxcr5, Cxcr6, Cxc/2, Cxcl9, Fpr2 \\
\hline Auto-Spleen & Down-regulated & Ccr7, Cxcl7, Ccr6, Ccr4, Sstr2, Penk, S1pr3, Npy, Hebp1, Flt3 \\
\hline Allo-Liver vs & Up-regulated & Kng1, Plg, Rdh7, Cps1, Serpinc1, Apof, Uox, Fga, Apoc3, Hpx \\
\hline Auto-Spleen & Down-regulated & Ccr7, Cxcr4, Ccr3, Ccr4, Htr1b, Ccr6, Cxcl7, s1pr3, Npy, Ptger3 \\
\hline Allo-Lung vs & Up-regulated & C3, Cxcl16, Ccl9, Ccr1, Cxcr2, Cxcl5, Cxcl3, Cxcl1, Ccr8, Cxcl10 \\
\hline Auto-Spleen & Down-regulated & Ccnb1, Cdc2O, Aurkb, Aurka, Plk1, Bub1b, Mcm2, Cdc6, Mcm5, Cdk1 \\
\hline
\end{tabular}

\section{Figures}


A
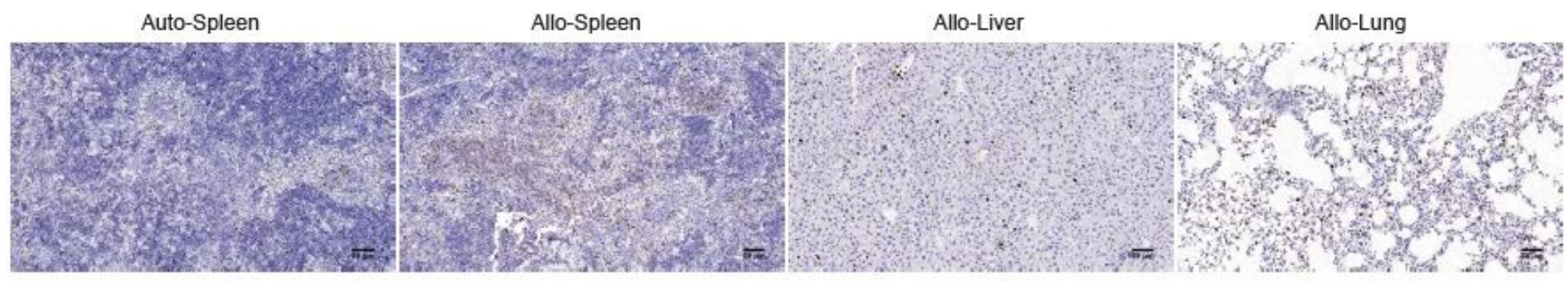

B

Auto-Spleen

Allo-Spleen
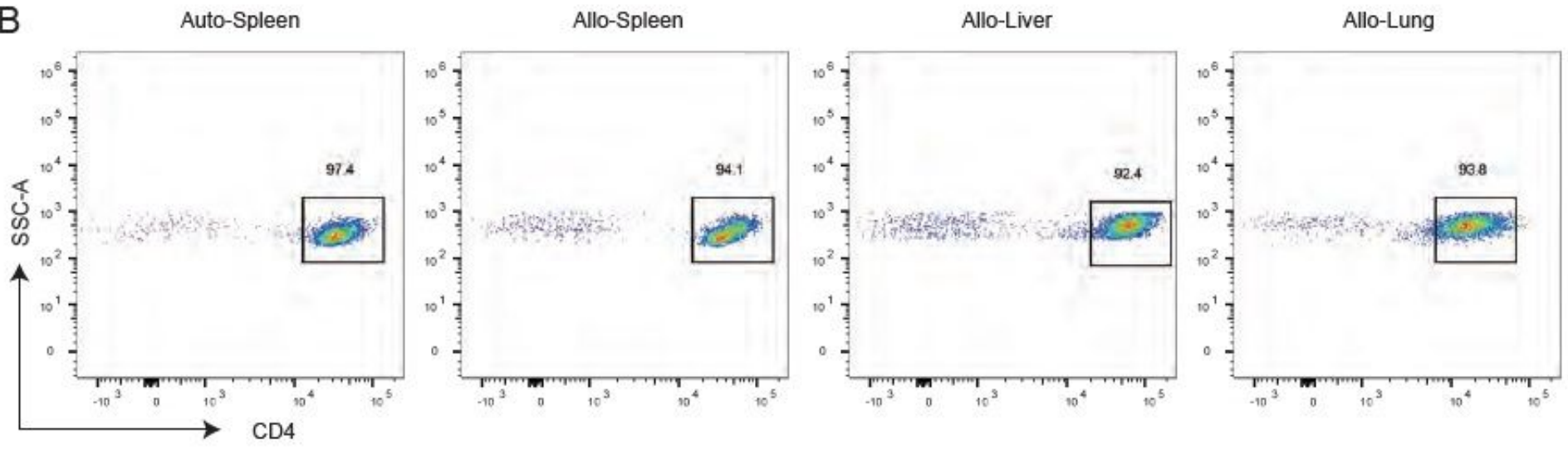

C
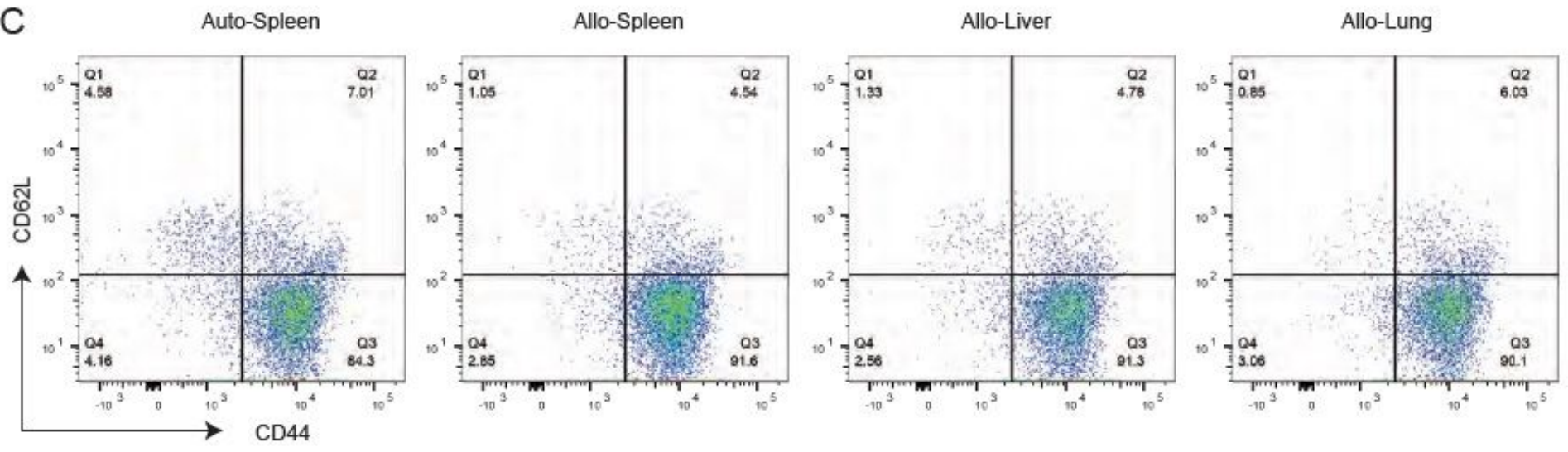

Figure 1

The ratio of the effector CD4+ T cells. (A) The organs stained with antibodies CD4. (B) Percent of CD4+ T cells in Auto-Spleen, Allo-Spleen, Allo-Liver and Allo-Lung. (C) Percent of the effector CD4+ T cells (CD4+CD44+CD62Llow) in Auto-Spleen, Allo-Spleen, Allo-Liver and Allo-Lung. The effector CD4+ T cells (CD4+CD44+CD62Llow) were detected by flow cytometry analysis. 


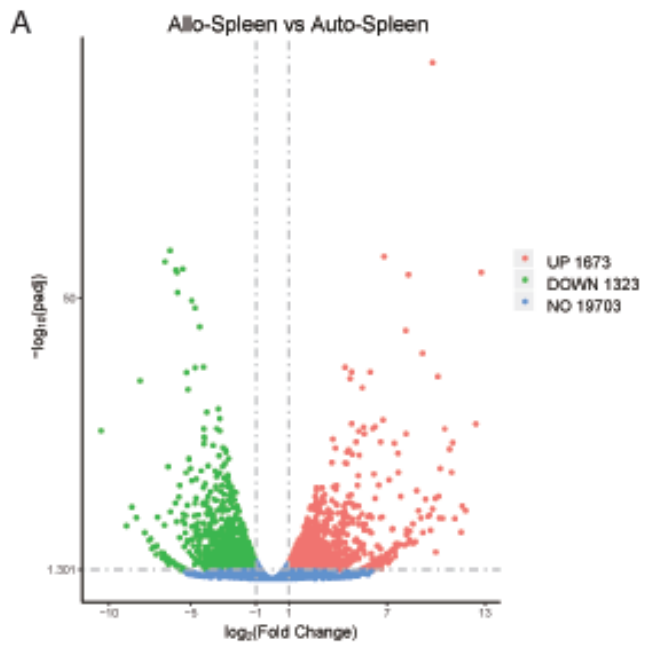

B
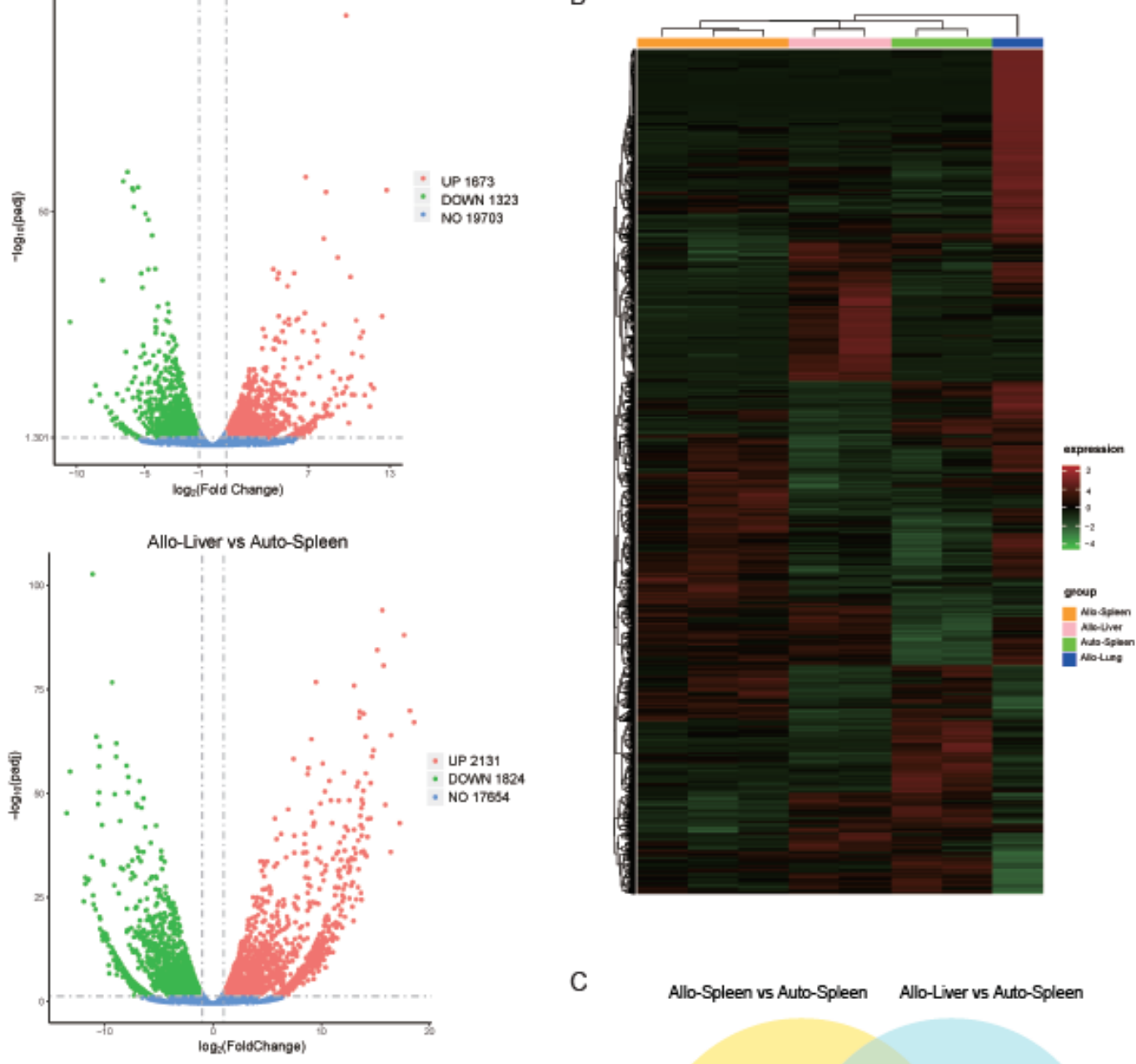

C
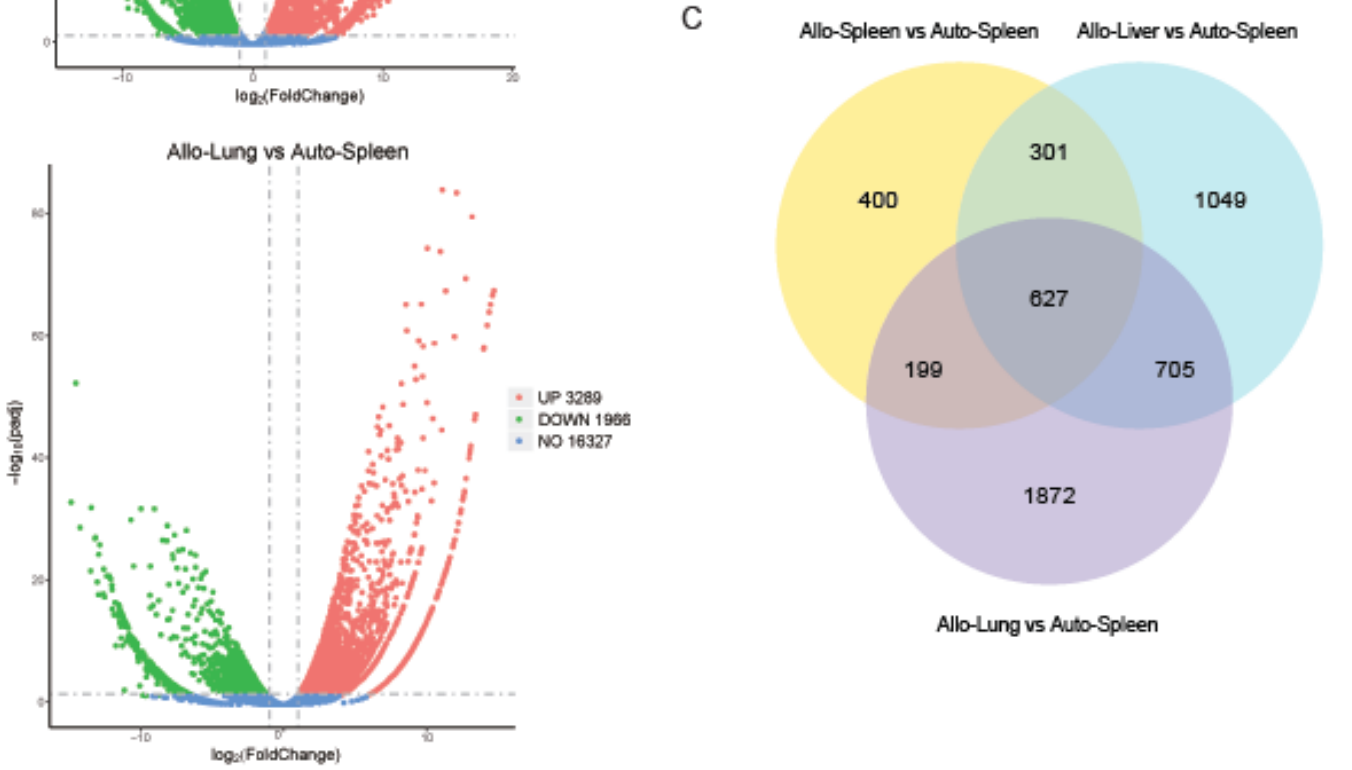

\section{Figure 2}

The DEGs among spleen, liver and lung. (A) Volcano map of DEGs. Log2(Fold Change>1) and padj<0.05 were consider significant. (B) The heatmap of DEGs. Each column represents a sample, each row represents a gene, and different expression multiples are represented by different colors. The red blocks are for high expression genes, while the green blocks are for low expression genes. (C) Venn of DEGs. 
A
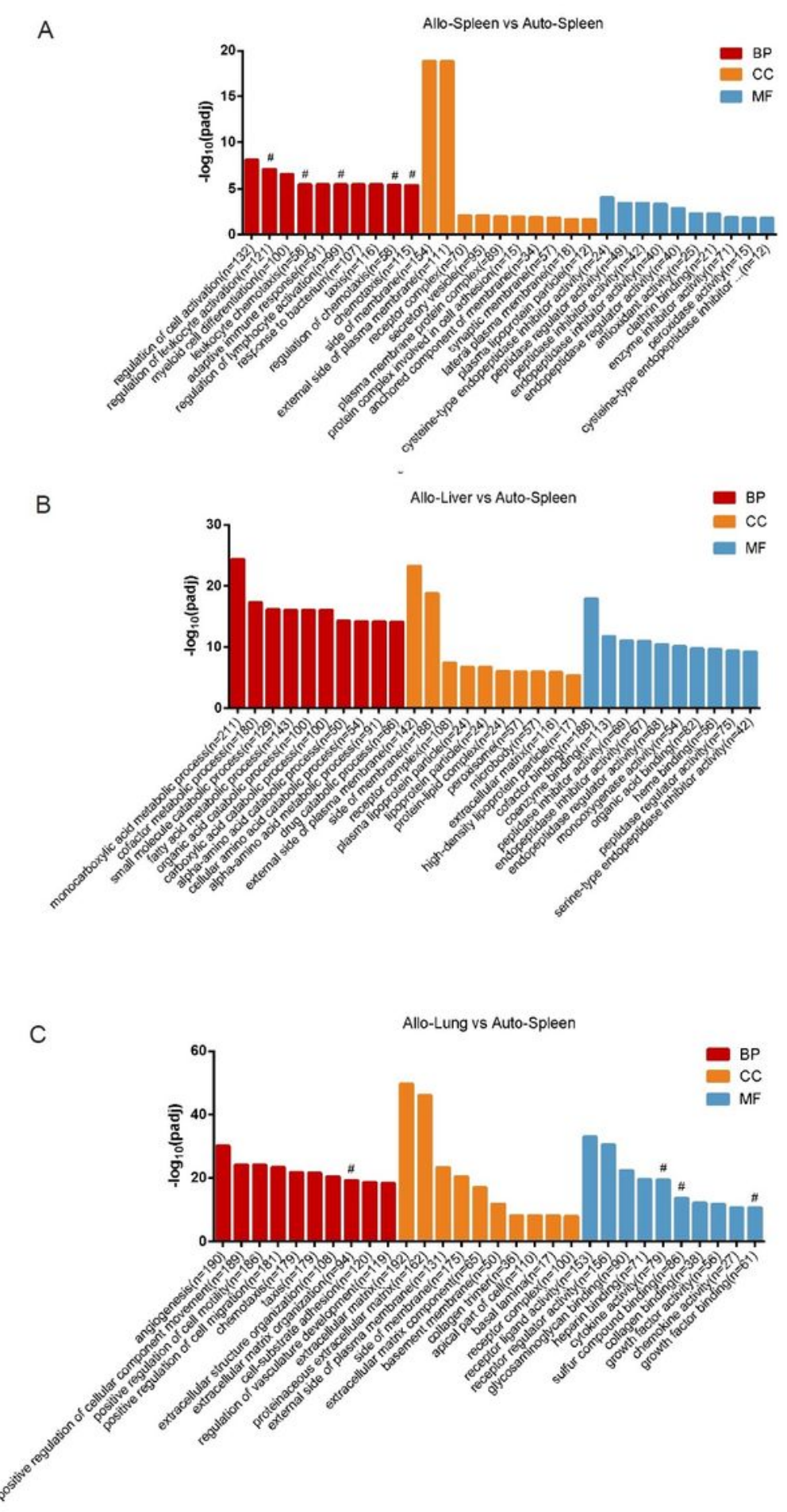

\section{Figure 3}

Enriched Gene Ontology (GO) terms. (A) The top 10 biological process (BP), cellular component (CC) and molecular function (MF) terms between Allo-Spleen and Auto-Spleen. (B) The top 10 BP, CC and MF terms between Allo-Liver and Auto-Spleen. (C) The top 10 BP, CC and MF terms between Allo-Lung and AutoSpleen. \# for GO terms associated with immunoregulation. 
A

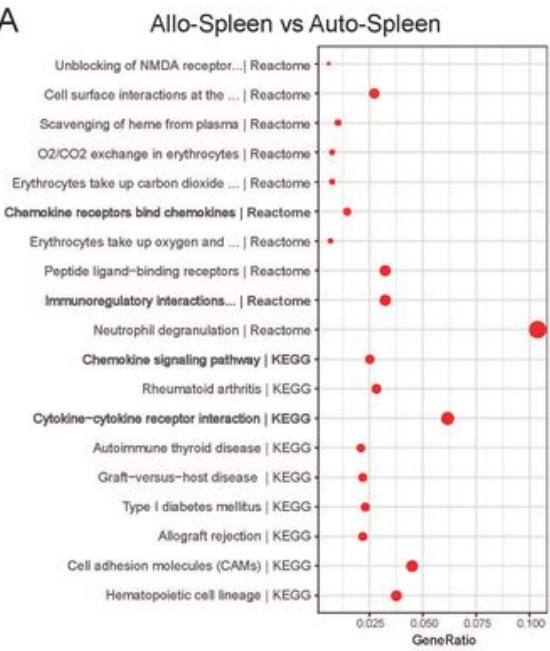

B

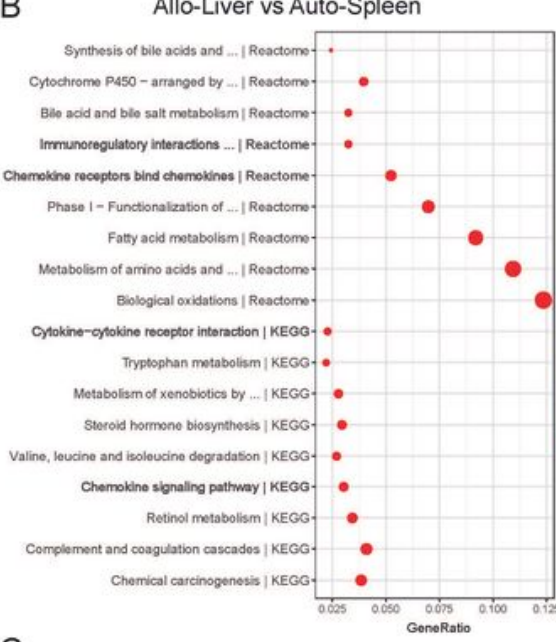

C

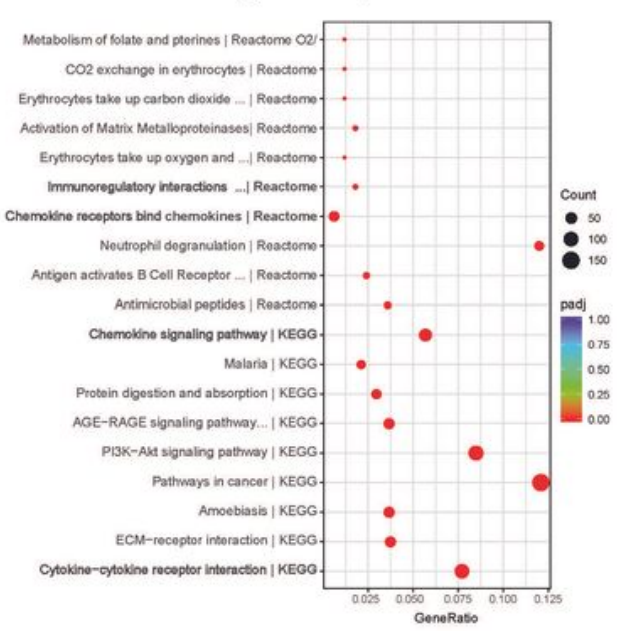

D
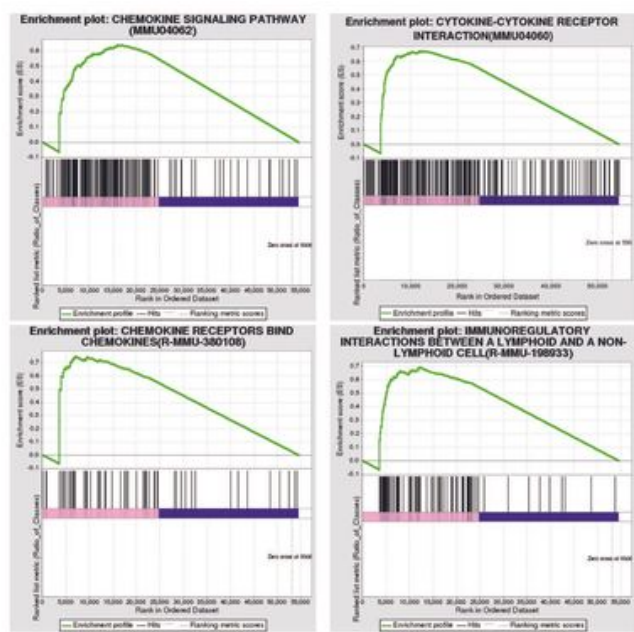

E
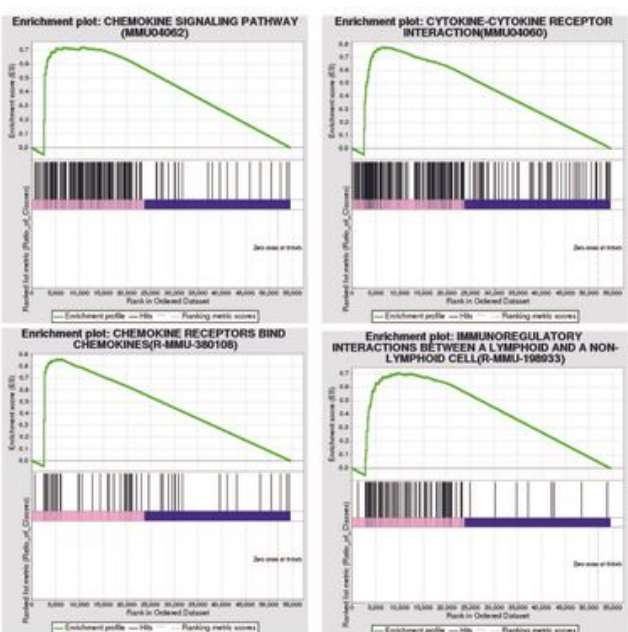

F
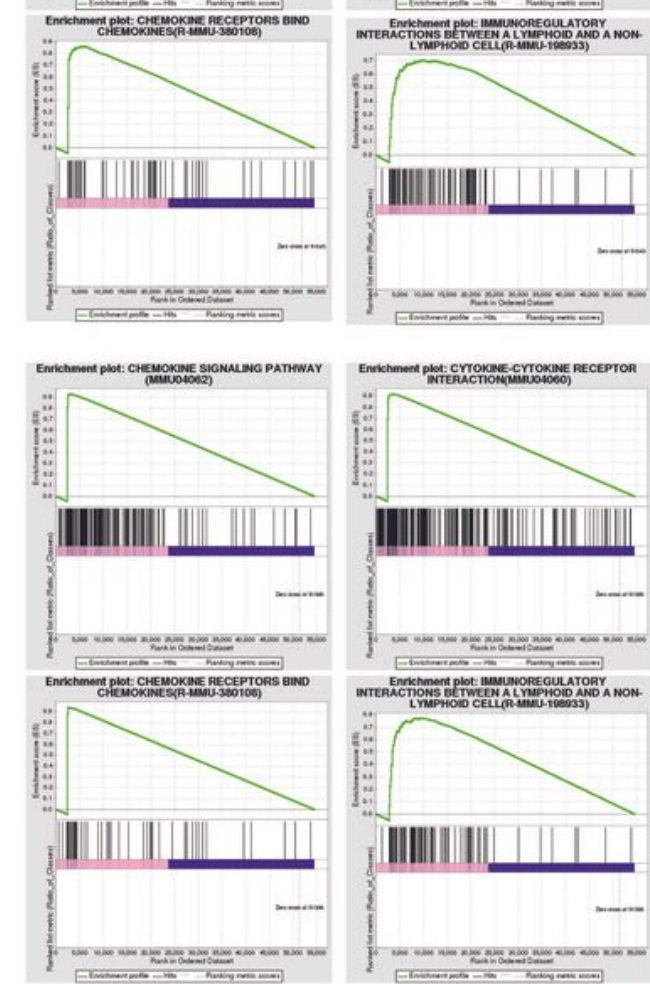

Figure 4

Enriched Kyoto Encyclopedia of Genes and Genomes (KEGG) and Reactome pathway terms. The top 10 KEGG and Reactome terms between Allo-Spleen and Auto-Spleen (A), Allo-Liver and Auto-Spleen (B), AlloLung and Auto-Spleen (C). The immune-related KEGG and Reactome pathway were analyzed using GSEA assays between Allo-Spleen and Auto-Spleen (D), Allo-Liver and Auto-Spleen (E), Allo-Lung and Auto- 
Spleen (F). The size of the dots represents the number of enrichment genes in the pathway. The different color of the dots indicates the different $p$ value.

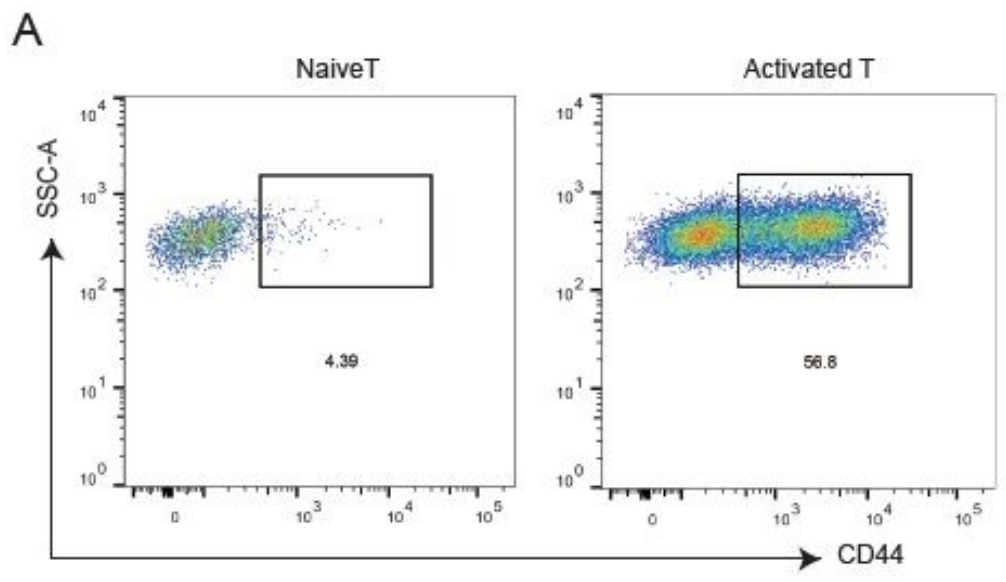

B
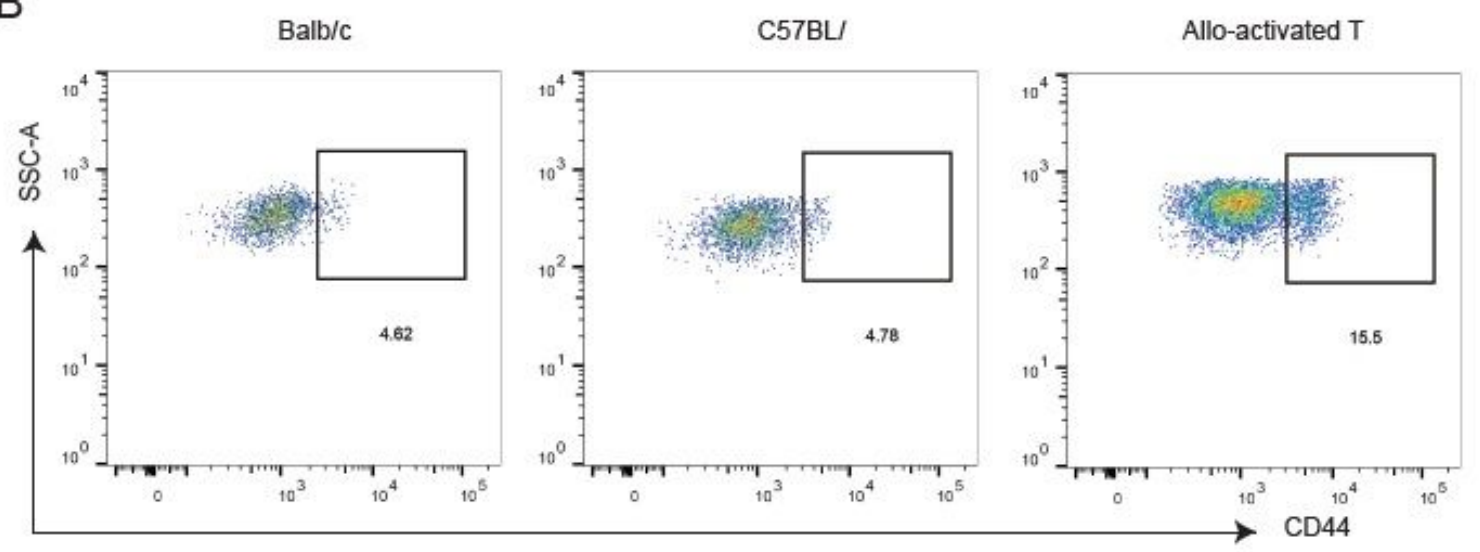

C

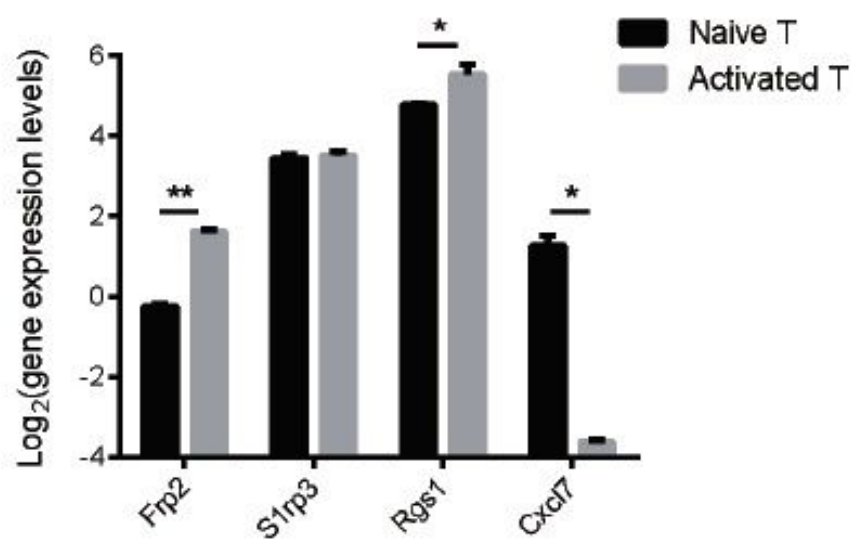

$\mathrm{D}$

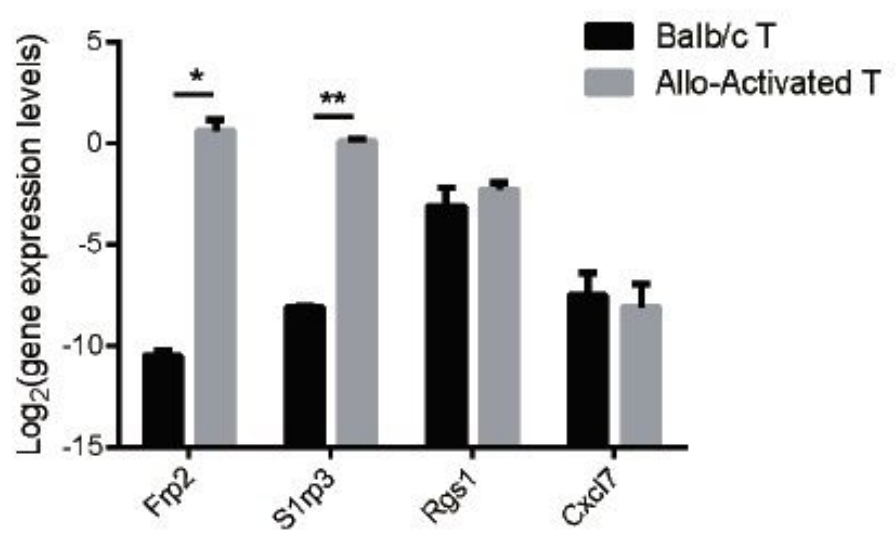

\section{Figure 5}

The verification of the expression of hub genes in virto. (A) The percent of activated CD4+ T cells in Naive $T$ and Activated $T$ cell by induction of anti-CD3/CD28 antibodies. (B) The percent of the activated CD4+ T cells in Balb/c, C57BL/ 6 and Allo-activated T cells after MLR. The activated CD4+ T cells (CD4+CD44+) were detected by flow cytometry analysis. (C) The expression levels of genes from activated T cells were detected.(D) The expression levels of genes from FACS-sorted activated CD4+ T cells were detected. The 
$Y$-axis is the log2 scale of the expression level of hub genes. Each experiment was performed at least three times and the results were presented as Mean \pm SD.

A

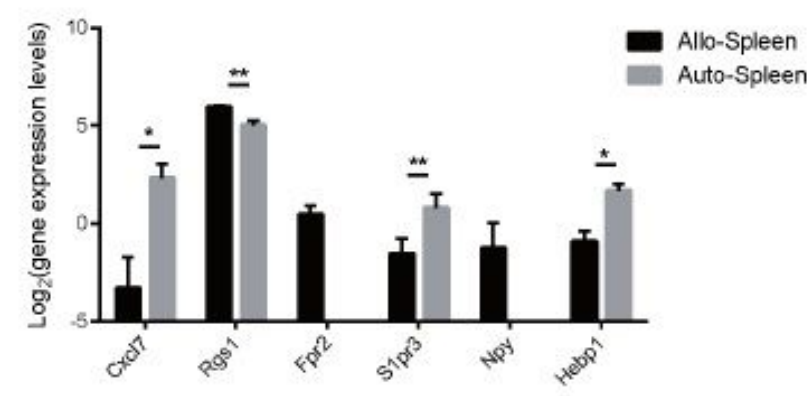

B
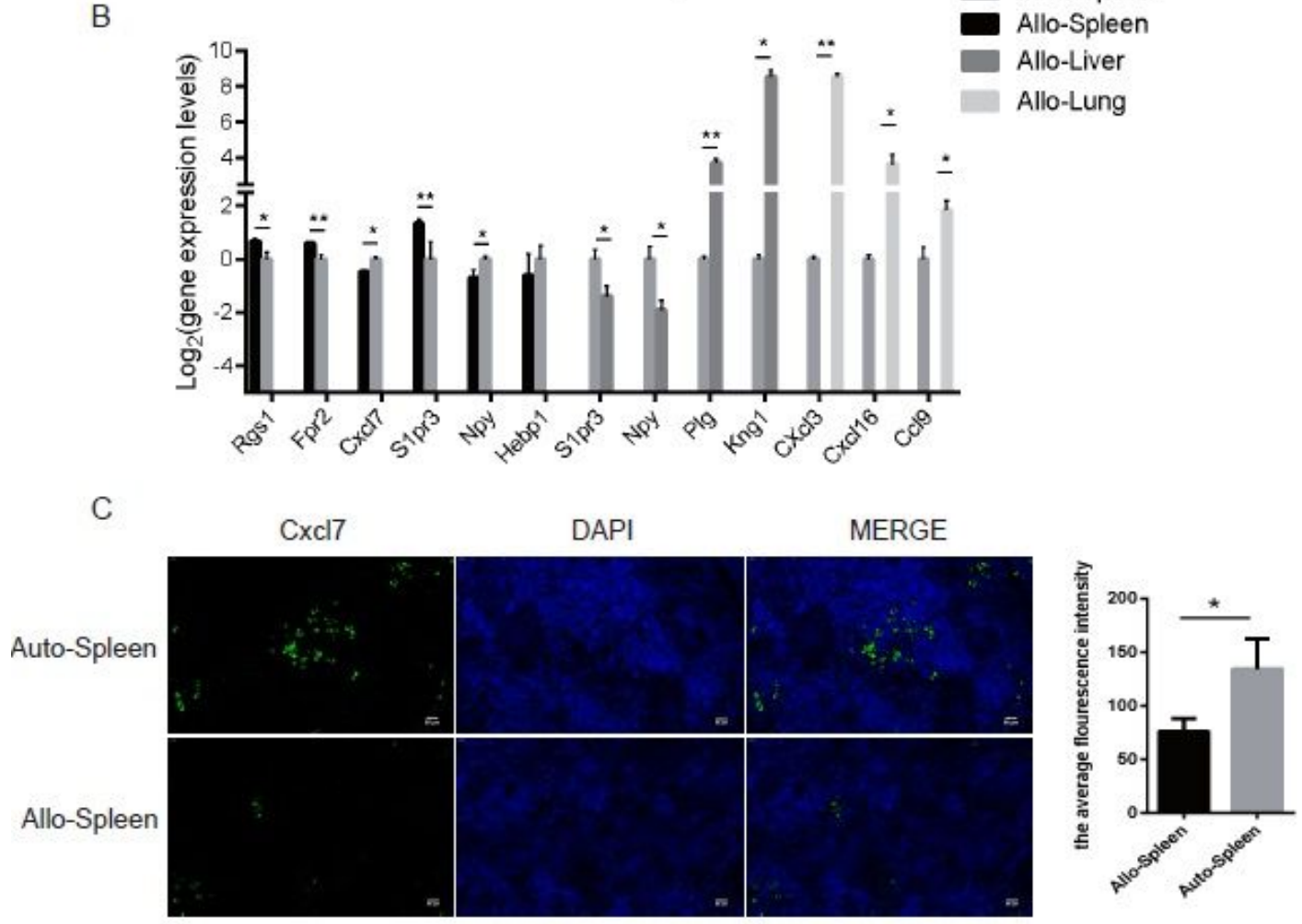

D
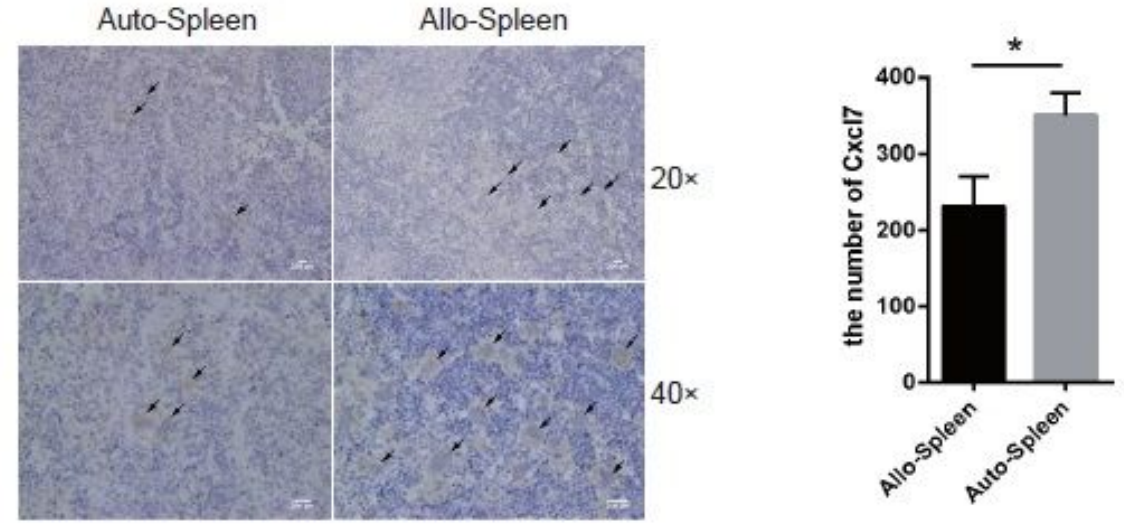

Figure 6

The verification of the expression of hub genes in vivo. (A)The hub genes from Allo-Spleen and AutoSpleen were verified in GSE126518. (B) The hub genes were verified from different tissues by qRT-PCR. CXCL7 deposition detected in target areas of spleen in aGVHD mouse animals. (C) Quantification of the 
histology staining shown. The left is one representative section per group. The right is the number of CXCL7 per scale in the spleen. CXCL7 were detected by colocalization of CXCL7 (green) and dapi(blue). CXCL7 deposition was quantified on a 0-3 scale to determine the amount of antibody in the tissues. (D)Representative spleen histology staining revealing the expression of CXCL7 (brown) $7 \mathrm{~d}$ after HSCT in Auto-HSCT and Allo-HSCT mice. Cells were stained with CXCL7 (brown). Bars, 50 or $200 \mu \mathrm{m}$. ${ }^{*}$ for $p<0.05$, ** for $p<0.01$. The $Y$-axis is the log 2 scale of the expression level of hub genes. Each experiment was performed at least three times and the results were presented as Mean \pm SD.

\section{Supplementary Files}

This is a list of supplementary files associated with this preprint. Click to download.

- SupplementaryFigures.pdf 